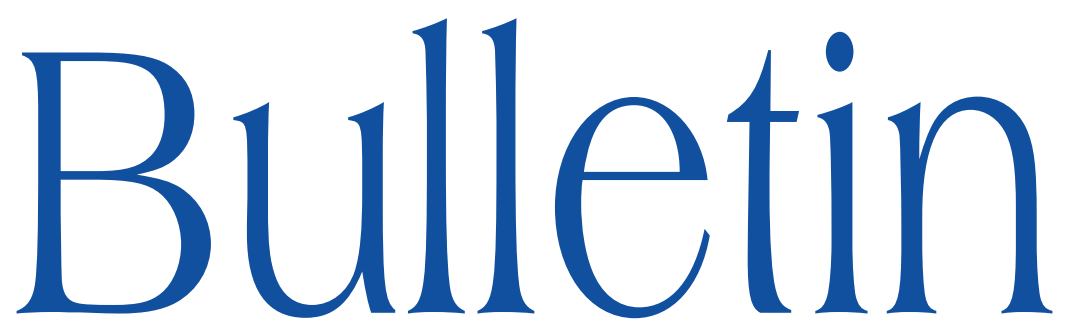

de la SOCIÉTÉ MATHÉMATIQUE DE FRANCE

\title{
TOPOLOGICAL DISJOINTNESS FROM ENTROPY ZERO SYSTEMS
}

Wen Huang \& Kyewon Koh Park \& Xiangdong Ye

Tome 135

Fascicule 2

2007 


\title{
TOPOLOGICAL DISJOINTNESS FROM ENTROPY ZERO SYSTEMS
}

\author{
By Wen Huang, Kyewon Koh Park \& Xiangdong Ye
}

\begin{abstract}
The properties of topological dynamical systems $(X, T)$ which are disjoint from all minimal systems of zero entropy, $\mathcal{M}_{0}$, are investigated. Unlike the measurable case, it is known that topological $K$-systems make up a proper subset of the systems which are disjoint from $\mathcal{M}_{0}$. We show that $(X, T)$ has an invariant measure with full support, and if in addition $(X, T)$ is transitive, then $(X, T)$ is weakly mixing. A transitive diagonal system with only one minimal point is constructed. As a consequence, there exists a thickly syndetic subset of $\mathbb{Z}_{+}$, which contains a subset of $\mathbb{Z}_{+}$ arising from a positive entropy minimal system, but does not contain any subset of $\mathbb{Z}_{+}$ arising from a zero entropy minimal system. Moreover we study the properties of topological dynamical systems $(X, T)$ which are disjoint from larger classes of zero entropy systems.
\end{abstract}

Texte reçu le 26 avril 2006, révisé le 28 septembre 2006

Wen Huang, University of Science and Technology of China, Department of Mathematics, Hefei, Anhui, 230026 (P.R. China) • E-mail : wenh@mail.ustc.edu.cn

Kyewon Кон Park, Ajou University, Department of Mathematics, Suwon 442-729 (Korea) E-mail : kkpark@madang.ajou.ac.kr

Xiangdong Ye, University of Science and Technology of China, Department of Mathematics, Hefei, Anhui, 230026 (P.R. China) • E-mail : yexd@ustc.edu.cn

2000 Mathematics Subject Classification. - 54H20.

Key words and phrases. - Disjointness, minimality, entropy, density.

The first and third authors are supported by SRFDP 20050358053 and NSFC Grants 10531010 and 10401031 . The first author is supported by NECT. The second author is supported in part by KRF Grant R05-2004-000-108640-0. The third author is supported by FANEDD200520. 
RÉSumÉ (Disjonction topologique des systèmes d'entropie nulle)

Nous étudions les propriétés des systèmes topologiques dynamiques $(X, T)$ qui sont disjoints de tout système minimal d'entropie nulle $\mathcal{M}_{0}$. Contrairement au cas mesurable, il est connu que les $K$-systèmes topologiques constituent un sous-ensemble propre des systèmes disjoints de $\mathcal{M}_{0}$. Nous montrons que $(X, T)$ a une mesure invariante à support plein, et que si, de plus, $(X, T)$ est transitif alors il est faiblement mélangeant. Nous construisons un système diagonal transitif avec un seul point minimal. Par conséquent, il existe un sous-ensemble grassement syndétique de $\mathbb{Z}_{+}$, qui contient un sous-ensemble de $\mathbb{Z}_{+}$, provenant d'un système minimal d'entropie positive, mais qui ne contienne aucun sous-ensemble de $\mathbb{Z}_{+}$provenant d'un système minimal d'entropie nulle. D'autre part, nous étudions les propriétés des systèmes topologiques dynamiques $(X, T)$ qui sont disjoints de classes plus larges de systèmes à entropie nulle.

\section{Introduction}

By a topological dynamical system (TDS for short) $(X, T)$, we mean a compact metric space $X$ with a continuous surjective map $T$ from $X$ to itself. A measurable system is defined to be K-mixing or a K-system if and only if every nontrivial partition has positive entropy. Equivalently a system is K-mixing if and only if it has no nontrivial entropy zero factor. It is well known that if a system is K-mixing, then it is strongly mixing. Entropy pairs, uniformly positive entropy systems (u.p.e.) and completely positive entropy systems (c.p.e.) have been introduced by Blanchard [1], [2] in search for the topological definition of a $K$-system. We define a system is topologically $K$ if and only if every nontrivial finite open cover (each element is not dense) has positive entropy. It is known [11] that if a system is topologically $\mathrm{K}$ and minimal, then it is topologically strongly mixing (it is still an open question that minimality and u.p.e. imply strongly mixing property).

In [5] the notion of disjointness was defined both in topological and measuretheoretical settings. If $(X, T)$ and $(Y, S)$ are two TDS we say $J \subset X \times Y$ is a joining of $X$ and $Y$ if $J$ is a non-empty closed invariant set and is projected onto $X$ and $Y$ respectively. If there is only one obvious joining $J=X \times Y$, we then say that $(X, T)$ and $(Y, S)$ are disjoint or $(X, T) \perp(Y, S)$. Let $\mathcal{M}$ be the collection of all minimal systems. As the disjointness of two TDS implies one of them is minimal, thus this naturally leads us to a question to characterize $\mathcal{M}^{\perp}$ (Question E in [5]). It is known [5] that a weakly mixing system with a dense set of periodic points is in $\mathcal{M}^{\perp}$. Recently, the authors [13] have shown that there are transitive systems in $\mathcal{M}^{\perp}$ with no periodic points. Moreover, it is proved that if $(X, T) \in \mathcal{M}^{\perp}$ then $(X, T)$ must have a dense set of minimal points, and if in addition $(X, T)$ is transitive it must be weakly mixing. 
In ergodic theory, a measurable dynamical system (MDS, for short) is a $K$ system if and only if it is disjoint from any MDS of zero entropy. One of the motivations of the paper is to see if the above result can be extended to TDS. Note that if two non-trivial TDS are disjoint then one of them is minimal [5] and the other one has a dense set of recurrent points [13]. Hence when we consider disjointness from zero entropy systems, we assume the class of zero entropy systems is either minimal or transitive. In this paper we consider both cases.

When the class consists of minimal zero entropy systems (denoted by $\mathcal{M}_{0}$ ), we prove that if $(X, T) \perp \mathcal{M}_{0}$, then $(X, T)$ has an invariant measure with full support, and if in addition $(X, T)$ is transitive, $(X, T)$ is weakly mixing. Note that it has been proved that transitive diagonal systems which include u.p.e. systems are in $\mathcal{M}_{0}^{\perp}$ [2]. When the class consists of transitive zero entropy systems with invariant measures of full support (denoted by $E_{0}$ ), we prove that if $(X, T) \perp E_{0}$, then $(X, T)$ is minimal and has c.p.e. We remark that systems which are disjoint from all transitive zero entropy systems are the trivial ones. If we restrict ourselves to a subclass $M_{0}$ (transitive zero entropy TDS with a dense set of minimal points) of $E_{0}$, we can show that all minimal diagonal systems are in $M_{0}^{\perp}$. It is an open question if there is a TDS in $M_{0}^{\perp} \backslash E_{0}^{\perp}$.

We construct a transitive diagonal system with only one minimal point. One consequence is that there is a TDS in $\mathcal{M}_{0}^{\perp} \backslash \mathcal{M}^{\perp}$. Another consequence is that there exists a thickly syndetic subset of $\mathbb{Z}_{+}$, which contains a subset of $\mathbb{Z}_{+}$arising from a positive entropy minimal system, but does not contain any subset of $\mathbb{Z}_{+}$arising from a zero entropy minimal system.

Now we recall some of the definitions.

- We say that $(X, T)$ is transitive if for each pair of non-empty open subsets $U$ and $V, N(U, V)=\left\{n \in \mathbb{Z}_{+}: T^{-n} V \cap U \neq \varnothing\right\}$ is infinite, where $\mathbb{Z}_{+}=\{0\} \cup \mathbb{N}$.

- $(X, T)$ is weakly mixing if $(X \times X, T \times T)$ is transitive.

- We say that $x \in X$ is a transitive point if its orbit orb $(x, T)=$ $\{x, T(x), \ldots\}$ is dense in $X$. It is well known that if $(X, T)$ is transitive then the set of transitive points is a dense $G_{\delta}$ set (denoted by $\operatorname{Tran}_{T}$ ) and if $\operatorname{Tran}_{T}=X$ we say that $(X, T)$ is minimal.

- For a TDS $(X, T)$, we call $x \in X$ a minimal point if $(\operatorname{cl}(\operatorname{orb}(x, T)), T)$ is a minimal subsystem of $(X, T)$. It is well known that $x$ is a minimal point of $(X, T)$ if and only if for any neighborhood $U$ of $x$,

$$
N(x, U):=\left\{n \in \mathbb{Z}_{+}: T^{n} x \in U\right\}
$$

is syndetic, i.e. with bounded gaps.

We would like thank the referee for the helpful remarks which made the paper much easier to follow. 


\section{TDS with an invariant measure of full support}

In this section, we study the properties of $(X, T)$ which is disjoint from the class $E_{0}$. Recall that a TDS $(X, T)$ is an E-system if it is transitive and has an invariant measure $\mu$ with full support, i.e., $\operatorname{supp}(\mu)=X$; it is an $M$-system if it is transitive and the set of minimal points is dense; and it is topologically ergodic (TE, for short) if $(X, T)$ is transitive and for each pair of non-empty open subsets $U, V$ of $X, N(U, V)$ is syndetic. It is known that a minimal system is an $E$-system, and an $E$-system is TE [9]. However there exist a TEsystem which is not an $E$-system, and an $E$-system which is not minimal. Two TDS are weakly disjoint if their product is transitive. Note that if both $(X, T)$ and $(Y, S)$ are transitive and $(X, T) \perp(Y, S)$, then they are weakly disjoint.

Since we need some of the results in [13], we summarize them in the following proposition.

Proposition 2.1. - Let $(X, T)$ be a TDS.

1. If $(X, T) \perp(Y, S)$ with $(Y, S)$ minimal and non-trivial, then $R(T)$ is a dense $G_{\delta}$ set of $X$, where

$$
R(T)=\left\{x \in X: \text { there is } n_{i} \rightarrow+\infty \text { with } T^{n_{i}} x \rightarrow x\right\}
$$

is the set of all recurrent points of $(X, T)$.

2. Let $T$ be invertible, $V$ be a non-empty open set of $X$ and

$$
Y=\operatorname{cl}\left(\bigcup_{i \in \mathbb{Z}} T^{i} V\right) .
$$

If $(X, T) \perp \mathcal{M}$, then $(Y, T) \perp \mathcal{M}$.

3. If there are transitive sub-systems $\left(X_{i}, T\right)$ of $(X, T)$ satisfying that $\cup_{i} X_{i}$ is dense in $X$ and $\left(X_{i}, T\right) \perp \mathcal{M}$ for each $i \in \mathbb{N}$. Then $(X, T) \perp \mathcal{M}$.

4. Let $(X, T)$ be an equicontinuous system. If $(X, T) \in \mathcal{M}^{\perp}$ then each point of $X$ is a fixed point. Consequently, if $(Y, S)$ is TDS with $(Y, S) \in \mathcal{M}^{\perp}$, then the maximal equicontinuous factor of $(Y, S)$ consists of fixed points.

5. If $(X, T) \in \mathcal{M}^{\perp}$, then $(X, T)$ has dense minimal points. If in addition, $(X, T)$ is transitive, then $(X, T)$ is weakly mixing.

According to Proposition 2.11), when considering our question of disjointness from entropy zero systems, it is natural to restrict our attention to TDS with dense sets of recurrent points and having zero entropy. The following proposition tells us that this class is too large. Before we go on to prove the proposition, let us recall some more definitions. Let $S$ be a subset of $\mathbb{Z}_{+}$. The upper Banach density of $S$ is

$$
B D^{*}(S)=\limsup _{|I| \rightarrow+\infty} \frac{|S \cap I|}{|I|},
$$


where $I$ ranges over intervals of $\mathbb{Z}_{+}$, while the upper density of $S$ is

$$
D^{*}(S)=\limsup _{n \longrightarrow \infty} \frac{|S \cap[1, n]|}{n} .
$$

Lower Banach density is defined analogously and it is easy to see that the lower Banach density of $S$ is one if for each $a<1$ there is $N$ such that for any subinterval $I$ of $\mathbb{Z}_{+}$with $|I| \geq N$ we have $|S \cap I| \geq a|I|$. Denote the set of subsets of $\mathbb{Z}_{+}$with lower Banach density one by $\mathcal{F}_{\text {lbd } 1}$.

A subset of $\mathbb{N}$ is an $I P$-set if it is equal to some

$$
F S\left(\left\{p_{i}\right\}_{i=1}^{\infty}\right)=\left\{p_{i_{1}}+p_{i_{2}}+\cdots+p_{i_{k}}: k \in \mathbb{N}, 1 \leq i_{1}<i_{2}<\cdots<i_{k}\right\},
$$

where $p_{i} \in \mathbb{N}$. A subset of $\mathbb{N}$ is called an $I P^{*}$-set, if it has no-empty intersection with any IP-set.

For any $S \subset \mathbb{Z}_{+}$let $1_{S}$ denote the indicator function from $\mathbb{Z}_{+}$to $\{0,1\}$, i.e. $1_{S}(s)=1$ if $s \in S$ and $1_{S}(s)=0$ if $s \notin S$. Let $\Sigma_{2}=\{0,1\}^{\mathbb{Z}_{+}}, \sigma$ be the shift and $[1]=\left\{x \in \Sigma_{2}: x(0)=1\right\}$.

Note that the topological entropy of a TDS $(X, T)$ is denoted by $h_{\text {top }}(X, T)$. The main reason why the following proposition holds is that each $I P$-set contains an $I P$-subset with zero upper Banach density.

Proposition 2.2. - If $(X, T)$ is disjoint from all transitive zero entropy systems that do not have invariant measures with full support (TZNE for short), then $(X, T)$ is trivial.

Proof. - It is not hard to show that there exists a non-minimal weakly mixing TDS with a unique ergodic measure whose support is a singleton [10]. We note that this example is a non-minimal transitive zero entropy system without an invariant measure of full support. Hence $(X, T)$ is minimal. Let $x \in X$ and $U$ be an open neighborhood of $x$. Assume $(Y, S) \in$ TZNE, $y$ is a transitive point and $V$ is a non-empty open subset of $Y$. It is easy to see that

$$
J=\operatorname{cl}((\operatorname{orb}(x, y), T \times S)
$$

is a joining of $(X, T)$ and $(Y, S)$. Thus $J=X \times Y$, since $X \perp Y$. Particularly, $N(x, U) \cap N(y, V) \neq \varnothing$. We have:

Claim. - $N(x, U)$ is an $I P^{*}$-set.

Proof of claim. - Let $A$ be an IP-set generated by $p_{1}, p_{2}, \ldots$ Take $0=i_{0}<$ $i_{1}<i_{2}<\cdots$ such that $q_{1}>2$ and

$$
q_{j+1}>\left((j+1) 2^{j+1}+1\right) \sum_{k=1}^{j} q_{k}
$$


for $j=1,2, \ldots$, where $q_{j}=\sum_{k=i_{j-1}+1}^{i_{j}} p_{k}, j=1,2, \ldots$ Put

$$
A^{\prime}=\left\{q_{j_{1}}+q_{j_{2}}+\cdots+q_{j_{k}}: 1 \leq j_{1}<j_{2}<\cdots<j_{k}, k \in \mathbb{N}\right\} .
$$

Then $A^{\prime} \subset A$ and $A^{\prime}$ is also an IP-set. Now we show that

$$
B D^{*}\left(A^{\prime}\right)=\limsup _{|I| \rightarrow+\infty} \frac{\left|A^{\prime} \cap I\right|}{|I|}=0 .
$$

For this purpose, we take $a_{k}<b_{k}$ with $\lim _{k \rightarrow+\infty} b_{k}-a_{k}=+\infty$ and

$$
\lim _{k \rightarrow+\infty} \frac{\left|A^{\prime} \cap\left[a_{k}, b_{k}-1\right]\right|}{b_{k}-a_{k}}=B D^{*}\left(A^{\prime}\right) .
$$

Without loss of generality, we assume that $\left|A^{\prime} \cap\left[a_{k}, b_{k}-1\right]\right| \geq 2$ for each $k \in \mathbb{N}$.

For $k \in \mathbb{N}$, set

$$
m_{k}=\min \left\{A^{\prime} \cap\left[a_{k}, b_{k}-1\right]\right\} \quad \text { and } \quad M_{k}=\max \left\{A^{\prime} \cap\left[a_{k}, b_{k}-1\right]\right\} .
$$

As $m_{k}, M_{k} \in A^{\prime}$, we can assume $m_{k}=q_{i_{1}}+\cdots+q_{i_{u}}+\left(q_{s_{1}}+\cdots+q_{s_{w}}\right)$ and $M_{k}=q_{j_{1}}+\cdots+q_{j_{v}}+\left(q_{s_{1}}+\cdots+q_{s_{w}}\right)$, where $0 \leq i_{1}<i_{2}<\cdots<i_{u}<s_{1}<$ $\cdots<s_{w}, 0<j_{1}<j_{2}<\cdots<j_{v}<s_{1}<\cdots<s_{w}$ and $j_{v}>i_{u}$ (we put $q_{0}=0$ ). It is easy to see that for any $a \in A^{\prime} \cap\left[a_{k}, b_{k}-1\right]$,

$$
a=q_{k_{1}}+\cdots q_{k_{w}}+\left(q_{s_{1}}+\cdots+q_{s_{w}}\right),
$$

where $0 \leq k_{1}<k_{2}<\cdots<k_{w} \leq j_{v}$. Hence

$$
\left|A^{\prime} \cap\left[a_{k}, b_{k}-1\right]\right| \leq 2^{j_{v}+1} \text {. }
$$

As $q_{1}>2$ and $q_{j+1}>\left((j+1) 2^{j+1}+1\right)\left(\sum_{k=1}^{j} q_{k}\right)$ for $j=1,2, \ldots$, we get

$$
q_{j_{v}}-\sum_{l=1}^{j_{v}-1} q_{\ell} \geq j_{v} 2^{j_{v}}
$$

Moreover $b_{k}-a_{k} \geq q_{j_{v}}-\sum_{\ell=1}^{j_{v}-1} q_{\ell} \geq j_{v} 2^{j_{v}}$. Combining this fact and (1), we have

$$
\frac{\left|A^{\prime} \cap\left[a_{k}, b_{k}-1\right]\right|}{b_{k}-a_{k}} \leq \min \left\{\frac{2^{j_{v}+1}}{b_{k}-a_{k}}, \frac{2}{j_{v}}\right\} .
$$

Since $\lim _{k \rightarrow+\infty} b_{k}-a_{k}=+\infty$,

$$
B D^{*}\left(A^{\prime}\right) \leq \lim _{k \rightarrow+\infty} \min \left\{\frac{2^{j_{v}+1}}{b_{k}-a_{k}}, \frac{2}{j_{v}}\right\}=0 .
$$

Since $A^{\prime}$ is an IP-set, $1_{A^{\prime}} \in \Sigma_{2}$ is a recurrent point under the shift $\sigma$. Let

$$
Y=\operatorname{cl}\left(\operatorname{orb}\left(1_{A^{\prime}}, \sigma\right)\right) .
$$

Then $(Y, \sigma)$ is a transitive system and $Y \neq\{(0,0, \ldots)\}$. As $B D^{*}\left(A^{\prime}\right)=0,(Y, \sigma)$ has a unique $\sigma$-invariant measure $\delta_{(0,0,0, \ldots)}$. Hence $h_{\text {top }}(Y, \sigma)=0$ and $(Y, \sigma)$ does not have an invariant measure with full support. 
By the assumption, $(X, T) \perp(Y, \sigma)$. Moreover, one has

$$
N(x, U) \cap N\left(1_{A^{\prime}},[1]\right) \neq \varnothing .
$$

Since $N\left(1_{A^{\prime}},[1]\right)=A^{\prime}$, we get that $N(x, U) \cap A \supset N(x, U) \cap A^{\prime} \neq \varnothing$. As $A$ is arbitrary, $N(x, U)$ is an IP $^{*}$-set. This ends the proof of the claim.

According to the claim, $N(x, U)$ is an $\mathrm{IP}^{*}$-set for any open neighborhood of $x$. Thus, by [6, Thm. 9.11], $x$ is a distal point. As each point of $(X, T)$ is distal, $(X, T)$ is distal, and so $(X, T)$ has zero entropy (see [14]). Let $\left(Y^{\prime}, S^{\prime}\right)$ be a weakly mixing zero entropy system which is in TZNE and $Y^{\prime \prime}=Y^{\prime} \times X$. Then $Y^{\prime \prime}$ is in TZNE, and thus $X \perp Y^{\prime \prime}$. This implies that $X \perp X$, i.e., $X$ is trivial.

Thus when we consider disjointness with respect to zero entropy systems, we restrict our attention to systems admitting invariant measures with full support. The following lemma tells us that it is enough to restrict our attention to $E$ systems having zero entropy. We state the lemma in the case of zero entropy, although the proof is irrelevant of the entropy.

LEMmA 2.3. - If $(X, T)$ is a zero entropy TDS admitting an invariant measure with full support, then there are countably many transitive subsystems $\left\{X_{i}\right\}$ in $E_{0}$ such that their union is dense in $X$. Moreover, for a $\operatorname{TDS}(Y, S)$ if $X_{i} \perp Y$ for each $i$, then $X \perp Y$.

Proof. - Let $\left\{U_{i}\right\}$ be a countable bases of the topological space $X$ and $\mu$ in $M(X, T)$ with $\operatorname{supp}(\mu)=X$. Let

$$
\mu=\int_{\Omega} \theta \mathrm{d} m(\theta)
$$

be the ergodic decomposition of $\mu$. Since $\int_{\Omega} \theta\left(U_{i}\right) \mathrm{d} m(\theta)=\mu\left(U_{i}\right)>0$, for each $i$, there is an ergodic measure $\theta_{i} \in \Omega$ with $\theta_{i}\left(U_{i}\right)>0$. Let $G\left(\theta_{i}\right)$ be the set of generic points of $\theta_{i}$. It is well known that $\theta_{i}\left(G\left(\theta_{i}\right)\right)=1$. This implies that $\theta_{i}\left(G\left(\theta_{i}\right) \cap U_{i}\right)>0$. Thus there is $x_{i} \in G\left(\theta_{i}\right) \cap U_{i}$ which is a transitive point of $\left(\operatorname{supp}\left(\theta_{i}\right), T\right)$. Set $X_{i}=\operatorname{cl}\left(\operatorname{orb}\left(x_{i}, T\right)\right)$. Then $X_{i}$ is an $E$-system and their union is dense in $X$. It is easy to see that the entropy of $\left(X_{i}, T\right)$ is zero.

Furthermore, the last statement of the lemma is easy to prove.

REMARK 2.4. - For a TDS $(X, T)$ we can speak about maximal transitive subsystems. By the method in the proof of [13, Thm. 4.5] one can show that if $(X, T) \perp(Y, S)$, where $(Y, S)$ is a TDS with an invariant measure with full support and with zero entropy, then there exist countably many maximal transitive subsystems $\left\{Y_{i}\right\}$ in $E_{0}$ such that their union is dense in $Y$ and $X \perp Y_{i}$ for each $i$. 
The following theorem describes some sufficient conditions and some necessary conditions for a system in $E_{0}^{\perp}$. Recall that a TDS $(X, T)$ has c.p.e. if each nontrivial factor of $(X, T)$ has positive entropy. Note that we use $M(X, T)$ to denote the set of all Borel invariant probability measures of $(X, T)$.

Theorem 2.5. - If $X \perp E_{0}$, then $X$ is minimal and has c.p.e. If $X$ is minimal and for each $\mu \in M(X, T),\left(X, \mathcal{B}_{X}, \mu, T\right)$ is a measurable $K$-system, then $X \perp E_{0}$.

Proof. - Assume that $X \perp E_{0}$. Then it is clear that $X$ is minimal since there is a non-minimal system in $E_{0}$. Let $X^{\prime}$ be the maximal zero entropy factor of $X$ (see [4]). Then $X^{\prime} \perp E_{0}$ since disjointness is preserved by a factor. Particularly, $X^{\prime} \perp X^{\prime}$ since $X^{\prime} \in E_{0}$. This implies that $X^{\prime}$ is trivial, i.e. $X$ has c.p.e.

Now let $X$ be a minimal system such that for each $\mu \in M(X, T)$, $\left(X, \mathcal{B}_{X}, \mu, T\right)$ is a measurable $\mathrm{K}$-system, and let $(Y, S)$ be in $E_{0}$. Assume that $J$ is a joining of $(X, T)$ and $(Y, S)$. Let $\nu \in M(J, T \times S)$ have the property that $\pi_{Y}(\nu)=\nu^{\prime}$ has full support. We note that this is always possible because $(Y, S)$ is a factor of $(J, T \times S)$. Since a measurable $K$-system is disjoint from any zero entropy $\operatorname{MDS}$ (see [5]) and $\left(Y, \mathcal{B}_{Y}, \nu^{\prime}, S\right)$ has zero measurable entropy, $\nu^{\prime} \perp \pi_{X}(\nu)$. That is $\nu=\pi_{X}(\nu) \times \nu^{\prime}$. Since any invariant measure of a minimal system has full support, we have $\operatorname{supp}\left(\pi_{X}(\nu)\right)=X$, and thus $J \supseteq \operatorname{supp}(\nu)=\operatorname{supp}\left(\pi_{X}(\nu)\right) \times \operatorname{supp}\left(\nu^{\prime}\right)=X \times Y$. This implies that $J=X \times Y$ and hence $X \perp Y$.

REMARK 2.6. - It is well known that each ergodic measure-preserving system has a topological model which is strictly ergodic, i.e., for any ergodic system $(Y, \mathcal{D}, \nu, S)$ there is a $\operatorname{TDS}(X, T)$ with a unique invariant measure $\mu$ satisfying $\operatorname{supp}(\mu)=X$ and $\left(X, \mathcal{B}_{X}, \mu, T\right)$ is isomorphic to $(Y, \mathcal{D}, \nu, S)$. Now by Theorem 2.5 for any measurable $K$-system, its topological model which is strictly ergodic is disjoint from all elements of $E_{0}$.

Let $M$ be the class of $M$-systems, $M_{0}$ be the zero entropy class of $M$-systems and $\mathcal{M}_{0}$ be the class of minimal systems with zero entropy. To end the section we consider the properties of TDS in $M_{0}^{\perp}$.

Recall that for a $\operatorname{TDS}(X, T)$ a pair $\left(x_{1}, x_{2}\right) \in X \times X$ is an entropy pair if $x_{1} \neq x_{2}$ and for each closed disjoint neighborhood $U_{i}$ of $x_{i}$, the open cover $\left\{U_{1}^{c}, U_{2}^{c}\right\}$ has positive entropy. Let $E_{2}(X, T)$ denote the set of all entropy pairs. Then $E_{2}(X, T) \neq \varnothing$ iff $(X, T)$ has positive topological entropy; and that unless a factor map $\phi:(X, T) \rightarrow(Y, S)$ collapses an entropy pair $(x, y)$, the image pair $(\phi(x), \phi(y))$ is an entropy pair of $(Y, S)$ when $\phi(x) \neq \phi(y)$. 
Moreover, it is proved in [7], Theorem 3,5), that when $(X, T)$ and $(Y, S)$ are two TDS with $h_{\text {top }}(S)>0$ and $\mu \in M(X, T)$,

$$
E_{2}(X \times Y, T \times S) \supseteq\left\{\left(\left(x, y_{1}\right),\left(x, y_{2}\right)\right): x \in \operatorname{supp}(\mu),\left(y_{1}, y_{2}\right) \in E_{2}(Y, S)\right\} .
$$

We say that a $\operatorname{TDS}(X, T)$ has u.p.e. if

$$
E_{2}(X, T)=X \times X \backslash\{(x, x): x \in X\} .
$$

And a diagonal system is the one such that $E_{2}(X, T)$ contains

$$
\Delta^{1}=\{(y, T y): y \in X, y \neq T y\} .
$$

Note that u.p.e. implies diagonality and a transitive diagonal system has c.p.e. It is shown in [2] that a diagonal system is disjoint from all minimal systems with zero entropy.

TheOREM 2.7. - If $X$ is a minimal diagonal system, then $X \in M_{0}^{\perp}$. Also if $X \in M_{0}^{\perp}$, then $X$ is minimal and has c.p.e.

Proof. - First assume that $(X, T)$ is a minimal diagonal system. As $(X, T)$ is disjoint from all minimal systems with zero entropy [2], it is easy to see that $X \in M_{0}^{\perp}$, since by the definition, an $M$-system has a dense set of minimal subsystems.

Now let $(X, T) \in M_{0}^{\perp}$. Since there exists a non-minimal zero entropy system with dense set of minimal points, $(X, T)$ is minimal. Let $\left(X^{\prime}, R\right)$ be the maximal zero entropy factor of $(X, T)$. We have $\left(X^{\prime}, R\right) \in M_{0}^{\perp} \cap M_{0}$ and this implies that $X^{\prime}$ is trivial. Thus $X$ is minimal and has c.p.e.

We remark that there is an example which is minimal with c.p.e. but without u.p.e. [15]. To end the section we ask two natural questions:

1) Is there a minimal c.p.e. but not a diagonal system?

2) Is there a system in $M_{0}^{\perp} \backslash E_{0}^{\perp}$ ? In particular, is a minimal diagonal system in $E_{0}^{\perp}$ ?

\section{TDS disjoint from $\mathcal{M}_{0}$, the transitive case}

In this section, we consider the properties of systems which are in $\mathcal{M}_{0}^{\perp}$. A totally transitive TDS with a dense set of periodic points and zero entropy is described in [16], which is in $\mathcal{M}_{0}^{\perp}$ by [5]. Thus unlike in the measurable case, there are transitive TDS with zero entropy in $\mathcal{M}_{0}^{\perp}$.

According to [13] and [1], a totally transitive TDS with a dense set of regular minimal points and a transitive diagonal system are in $\mathcal{M}_{0}^{\perp}$. In this section we consider the necessary conditions. To answer our question, we transfer the problem to a problems of integers. That is, which subsets of $\mathbb{Z}_{+}$contain a subset that arises from a minimal system with zero entropy? 
Now we introduce the notion of a $z m$-set which plays an important role in solving our question.

Definition 3.1. - A subset $A$ of $\mathbb{Z}_{+}$is called an $z m$-set, if there exist a minimal zero entropy system $(Y, S), y \in Y$ and a non-empty open subset $V$ of $Y$ such that $A \supseteq N(y, V)$.

For a transitive system whether it is in $\mathcal{M}_{0}{ }^{\perp}$ can be checked via $z m$-sets as the following theorem shows.

THEOREM 3.2. - Let $(X, T)$ be a transitive system and $x \in$ Trans $_{T}$. Then $(X, T) \in \mathcal{M}_{0}^{\perp}$ if and only if $N(x, U) \cap A \neq \varnothing$ for any neighborhood $U$ of $x$ and any zm-set $A$.

Proof. - Let $(X, T) \in \mathcal{M}_{0}{ }^{\perp}$. For any $z m$-set $A$, there exist a minimal zero entropy system $(Y, S), y \in Y$ and a non-empty open subset $V$ of $Y$ such that $A \supseteq N(y, V)$.

Let $J=\operatorname{cl}(\operatorname{orb}((x, y), T \times S))$, then $J$ is a joining of $(X, T)$ and $(Y, S)$. Since $(X, T) \perp(Y, S), J=X \times Y$. Hence for any neighborhood $U$ of $x$ one has $N((x, y), U \times V) \neq \varnothing$. This implies $N(x, U) \cap A \neq \varnothing$.

Conversely, let $(Y, S)$ be a minimal zero entropy system and $J$ be a joining of $(X, T)$ and $(Y, S)$. It is clear that, there is $y \in Y$ with $(x, y) \in J$. For any neighborhood $U$ of $x$ and any non-empty open subset $V$ of $Y$, one has $N(x, U) \cap N(y, V) \neq \varnothing$ since $N(y, V)$ is a $z m$-set. This implies

$$
\operatorname{cl}(\operatorname{orb}((x, y), T \times S)) \cap(U \times V) \neq \varnothing .
$$

Since $\operatorname{cl}(\operatorname{orb}((x, y), T \times S))$ is $T \times S$-invariant and closed, one has

$$
\operatorname{cl}(\operatorname{orb}((x, y), T \times S))=X \times Y .
$$

Thus $J=X \times Y$.

Let $\mathcal{P}$ be the collection of all subsets of $\mathbb{Z}_{+}$. A subset $\mathcal{F}$ of $\mathcal{P}$ is a family, if it is hereditary upwards. That is, $F_{1} \subset F_{2}$ and $F_{1} \in \mathcal{F}$ imply $F_{2} \in \mathcal{F}$. Any subset $\mathcal{A}$ of $\mathcal{P}$ can generate a family $[\mathcal{A}]=\{F \in \mathcal{P}: F \supset A$ for some $A \in \mathcal{A}\}$.

Clearly, all $z m$-sets form a family, we call it the family of zm-sets. For any $S \subset \mathbb{Z}_{+}$let $1_{S}$ be the indicator function from $\mathbb{Z}_{+}$to $\{0,1\}$. Now, we have

Proposition 3.3. - The family of zm-sets is the family generated by

$$
\begin{array}{r}
\mathcal{F}_{m}^{\prime}=\left\{S \subset \mathbb{Z}_{+}: 1_{S} \text { is a minimal point of }\left(\Sigma_{2}, \sigma\right)\right. \text { and } \\
\left.\operatorname{cl}\left(\operatorname{orb}\left(1_{S}, \sigma\right)\right) \text { has zero entropy }\right\} .
\end{array}
$$


Proof. - Let $\mathcal{F}_{m}$ denote the family generated by $\mathcal{F}_{m}^{\prime}$. Clearly, if $1_{F}$ is the indicator function of $F$ then $F=N\left(1_{F},[1]\right)$. Hence $\mathcal{F}_{m}$ is contained in the family of $z m$-sets.

Now let $A$ be a $z m$-set. Then there exist a minimal zero entropy system $(Z, R), z_{0} \in Z$ and a non-empty open subset $W$ of $Z$ such that $A \supset N\left(z_{0}, W\right)$. It is well known that there exists a zero-dimensional, minimal and zero entropy system $(X, T)$ which is an extension of $(Z, R)$ (See for example [8, Prop. 2.4]). Let $\pi:(X, T) \rightarrow(Z, R)$ be the extension.

Take $x_{0} \in \pi^{-1}\left(z_{0}\right)$ and let $V^{\prime}=\pi^{-1}(W)$. It is easy to see that $A \supset$ $N\left(z_{0}, W\right) \supset N\left(x_{0}, V^{\prime}\right) \supset N\left(x_{0}, V\right)$, where $V$ is a clopen subset of $V^{\prime}$.

Then we do the classical lifting trick. Let

$$
\begin{aligned}
& Y=\left\{(x, t) \in X \times \Sigma_{2}: t(i)=1 \text { implies } T^{i} x \in V\right. \text { and } \\
& \left.\qquad(i)=0 \text { implies } T^{i} x \in X \backslash V\right\} .
\end{aligned}
$$

Then $Y$ is a $T \times \sigma$-invariant closed subset of $X \times \Sigma_{2}$. Let $\phi:(Y, T \times \sigma) \rightarrow(X, T)$ be the projection. Then $\left|\phi^{-1}(x)\right|=1$ for $x \in X$. That is, $\phi$ is an isomorphism, hence $(Y, T \times \sigma)$ is minimal and of zero entropy.

For $x_{0}$, there is a unique $t_{0} \in \Sigma_{2}$ such that $\left(x_{0}, t_{0}\right) \in Y$ and $t_{0}$ is the indicator function of $N\left(x_{0}, V\right)$. Projecting $Y$ to $\Sigma_{2}$ we see that $t_{0}$ is a minimal point of $\left(\Sigma_{2}, \sigma\right)$, and the closure of the orbit of $t_{0}$ has zero entropy. Hence $A \in \mathcal{F}_{m}$ as $A \supset N\left(x_{0}, V\right)$ and $t_{0}=1_{N\left(x_{0}, V\right)}$.

We will show that any subset of $\mathbb{Z}_{+}$in $\mathcal{F}_{\text {lbd } 1}$ contains a $z m$-set, where $\mathcal{F}_{\text {lbd1 }}$ denote the collection of subsets of $\mathbb{Z}_{+}$with lower Banach density one. To do so we need the following lemma. Note that if $\Omega$ is a finite symbolic set, $y \in \Omega^{\mathbb{Z}_{+}}$ and $k \in \mathbb{N}$, then we let $y[i ; i+k-1]=(y(i), y(i+1), \ldots, y(i+k-1)) \in \Omega^{k}$ and let

$$
\begin{aligned}
& N_{k}(y)=\#\left\{u \in \Omega^{k}: \exists i \in \mathbb{Z}_{+} \text {such that } u=y[i k ; i k+k-1]\right\} \\
& B_{k}(y)=\#\left\{u \in \Omega^{k}: \exists i \in \mathbb{Z}_{+} \text {such that } u=y[i ; i+k-1]\right\} .
\end{aligned}
$$

Lemma 3.4. - For $a \in \Omega$ and $y \in \Omega^{\mathbb{Z}_{+}}$let $F(y, a)=\left\{i \in \mathbb{Z}_{+}: y(i)=a\right\}$. If $F(y, a) \in \mathcal{F}_{\text {lbd1 }}$, then for any $\epsilon>0$ there exists $N>0$ such that $N_{k}(y)<2^{\epsilon k}$ for $k \geq N$.

Proof. - Let $\sigma$ be the shift map on $\Omega^{\mathbb{Z}_{+}}$and put $Y=\operatorname{cl}(\operatorname{orb}(y, \sigma))$. Since $F(y, a)$ belongs to $\mathcal{F}_{\mathrm{lbd} 1},(Y, \sigma)$ has a unique $\sigma$-invariant measure $\delta_{(a, a, a, \ldots)}$. Hence $h_{\text {top }}(Y, \sigma)=0$. Fix $\epsilon>0$. As

$$
\limsup _{k \rightarrow+\infty} \frac{\log N_{k}(y)}{k} \leq \lim _{k \rightarrow+\infty} \frac{\log B_{k}(y)}{k}=h_{\mathrm{top}}(Y, \sigma)=0,
$$

there exists $N>0$ such that $N_{k}(y)<2^{\epsilon k}$ for $k \geq N$. 
For a finite word $A \in \Omega^{k},|A|=k$ denotes the length of $A$. The following theorem is crucial for this section.

THEOREM 3.5. - If $F \in \mathcal{F}_{\mathrm{lbd} 1}$, then $F$ contains a zm-set.

Proof. - Let $F \in \mathcal{F}_{\text {lbd1 }}$. For each n, we will construct $y^{n}=1_{F_{n}} \in \Sigma_{2}$ for some

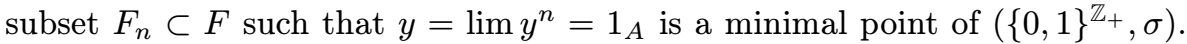
Let $Y=\operatorname{cl}(\operatorname{orb}(y, \sigma))$ and $[1]=\{x \in Y: x(0)=1\}$. We will show that $h_{\text {top }}(Y, \sigma)=0$. As $A \subset F$ and $A=N(y,[1])$ the theorem follows.

To obtain such a sequence $\left\{y^{n}\right\}$ we will construct a sequence $\left\{A_{n}\right\}$ of finite words on $\{0,1\}$ with the following properties:

1. $y^{n}$ begins with $A_{n}$ for each $n \in \mathbb{N}$;

2. $A_{i}$ appears in $y^{n}$ syndetically with gaps bounded by $\ell_{i}$ for each $i \leq n$;

3. $A_{n+1}$ begins with $A_{n}$ and

4. $N_{k_{i}}\left(y^{n}\right)<2^{k_{i} / i}$ for each $1 \leq i \leq n$, where $k_{i}=\left|A_{i}\right|$.

The reason we can do this is that $F \in \mathcal{F}_{\text {lbd1 }}$. More precisely we do as follows.

Step 1. - We will construct $A_{1}\left(k_{1}=\left|A_{1}\right|\right)$ and $F_{1} \subset F$ such that $A_{1}$ appears in $y^{1}=1_{F_{1}}$ with gaps bounded by $\ell_{1}, y^{1}$ begins with $A_{1}$ and $N_{k_{1}}\left(y^{1}\right)<2^{k_{1}}$.

Since $F \in \mathcal{F}_{\text {lbd } 1}$, there exists $K_{1}>0$ such that $N_{k}\left(1_{F}\right)<2^{k}$ for $k \geq K_{1}$ by Lemma 3.4. Put $k_{1}=\max \left\{K_{1}, \min F+1\right\}$ and $A_{1}=1_{F}\left[0 ; k_{1}-1\right]$. Then $N_{k_{1}}\left(1_{F}\right)<2^{k_{1}}$. Set

$$
W_{1}=\left\{i \in \mathbb{Z}_{+}:\left[i k_{1} ; i k_{1}+k_{1}-1\right] \subset F\right\} .
$$

Choose $y^{1} \in\{0,1\}^{\mathbb{Z}_{+}}$such that

$$
\begin{gathered}
y^{1}\left[0 ; k_{1}-1\right]=A_{1}, \quad y^{1}\left[i k_{1} ; i k_{1}+k_{1}-1\right]=A_{1}, i \in W_{1}, \\
y^{1}\left[j k_{1} ; j k_{1}+k_{1}-1\right]=1_{F}\left[j k_{1} ; j k_{1}+k_{1}-1\right] \text { if } j \notin W_{1} .
\end{gathered}
$$

Since $F \in \mathcal{F}_{\text {lbd1 } 1}$, we have $W_{1} \in \mathcal{F}_{\text {lbd1 }}$. Hence $W_{1}$ is syndetic with gaps bounded by some $\ell_{1}^{\prime}$. Put $\ell_{1}=\ell_{1}^{\prime} \cdot k_{1}$. Then $A_{1}$ appears in $y^{1}$ with gaps bounded by $\ell_{1}, F_{1} \subset F$ and $N_{k_{1}}\left(y^{1}\right) \leq N_{k_{1}}\left(1_{F}\right)<2^{k_{1}}$, where $1_{F_{1}}=y^{1}$.

Step 2. - Assume that $A_{1}, \ldots, A_{m}$ have been constructed and we will construct $A_{m+1}$ and $F_{m+1} \subset F$ such that

a) $A_{m+1}$ has the form of $A_{m} V_{m} A_{m}$ and if $k_{m+1}=\left|A_{m+1}\right|$ then $A_{m+1}=$ $y^{m}\left[0 ; k_{m+1}-1\right]$

b) $y^{m+1}\left[0 ; k_{m+1}-1\right]=A_{m+1}$ and $A_{i}$ appears in $y^{m+1}$ syndetically with gaps bounded by $\ell_{i}$ for each $1 \leq i \leq m+1$;

c) $F_{m+1}=\left\{i \in \mathbb{Z}_{+}: y^{m+1}(i)=1\right\} \subset F$;

d) $N_{k_{i}}\left(y^{m+1}\right)<2^{k_{i} / i}$ for each $1 \leq i \leq m+1$.

TOME $135-2007-\mathrm{N}^{\mathrm{O}} 2$ 
Since $W_{m} \in \mathcal{F}_{\mathrm{lbd} 1}$ and $A_{m}$ appears in $y^{m}$ at places $k_{m} \cdot W_{m}$ in $y^{m}$, there exists $K_{m+1}>0$ such that $N_{k \cdot k_{m}}\left(y^{m}\right)<2^{k /(m+1) \cdot k_{m}}$ for $k \geq K_{m+1}$ by Lemma 3.4.

Let $k_{m+1}^{\prime} \geq K_{m+1}$ with $k_{m+1}^{\prime} \in W_{m}$. Set $k_{m+1}=k_{m+1}^{\prime} \cdot k_{m}$ and let $A_{m+1}=y^{m}\left[0 ; k_{m+1}-1\right]$. Then $A_{m+1}$ has the form of $A_{m} V_{m} A_{m}$ and $N_{k_{m+1}}\left(y^{m}\right)<2^{k_{m+1} / m+1}$.

Set $W_{m+1}=\left\{i \in \mathbb{Z}_{+}:\left[i k_{m+1} ; i k_{m+1}+k_{m+1}-1\right] \subset F\right\}$.

Choose $y^{m+1} \in\{0,1\}^{\mathbb{Z}_{+}}$such that

$y^{m+1}\left[0 ; k_{m+1}-1\right]=A_{m+1}$,

$y^{m+1}\left[i k_{m+1} ; i k_{m+1}+k_{m+1}-1\right]=A_{m+1}, i \in W_{m+1}$ and

$y^{m+1}\left[i k_{m+1} ; i k_{m+1}+k_{m+1}-1\right]=y^{m}\left[i k_{m+1} ; i k_{m+1}+k_{m+1}-1\right], i \notin W_{m+1}$.

As $y^{m+1}$ and $y^{m}$ differ possibly only at $\left[i k_{m+1} ; i k_{m+1}+k_{m+1}-1\right], i \in W_{m+1}$, thus

$$
F_{m+1}=\left\{i \in \mathbb{Z}_{+}: y^{m+1}(i)=1\right\} \subset F_{m} \cup \bigcup_{i \in W_{m+1}}\left[i k_{m+1} ; i k_{m+1}+k_{m+1}-1\right] \subset F .
$$

As $F \in \mathcal{F}_{\text {lbd1 }}$, we have $W_{m+1} \in \mathcal{F}_{\text {lbd1 }}$. Hence $W_{m+1}$ is syndetic with gaps bounded by some $\ell_{m+1}^{\prime}$. Put $\ell_{m+1}=\ell_{m+1}^{\prime} \cdot k_{m+1}$. Then $A_{i}$ appears in $y^{m+1}$ with gaps bounded by $\ell_{i}$ and $N_{k_{i}}\left(y^{m+1}\right) \leq N_{k_{i}}\left(y^{m}\right)<2^{k_{i} / i}$ for each $1 \leq i \leq m+1$ by the construction.

In such a way for each $m \in \mathbb{N}$ we have defined a finite word $A_{m}$ and $y_{m} \in \Sigma_{2}$ satisfying 1)-4). Let $y=\lim A_{m}=\lim y^{m}$. By the construction, $A_{m}$ appears in $y$ with gaps bounded by $\ell_{m}$ for each $m \in \mathbb{N}$. That is, $y$ is a minimal point for the shift. It is obvious that $y \neq(0,0, \ldots)$. Let $Y=\operatorname{cl}(\operatorname{orb}(y, \sigma))$ and $U=[1]$. Then

$$
\varnothing \neq N(y, U)=\bigcup_{i=1}^{\infty}\left\{i \in \mathbb{Z}_{+}: A_{n}(i)=1,0 \leq i \leq k_{n}-1\right\} \subset \bigcup_{i=1}^{\infty} F_{n} \subset F .
$$

As $N_{k_{i}}\left(y^{m}\right)<2^{k_{i} / i}$ for $m \in \mathbb{N}, i=1,2, \cdots, m$, one has $N_{k_{i}}(y) \leq 2^{k_{i} / i}, i \in \mathbb{N}$.

For each $u \in B_{k}(y)$, there are $u_{1}, u_{2} \in N_{k}(y)$ such that $u<u_{1} u_{2}$. Thus $B_{k}(y) \leq k N_{k}(y)^{2}$ for any $k \in \mathbb{N}$ and hence

$$
\begin{aligned}
h_{\mathrm{top}}(Y, \sigma) & =\lim _{i \rightarrow+\infty} \frac{1}{k_{i}} \log B_{k_{i}}(y) \\
& \leq \lim _{i \rightarrow+\infty} \frac{1}{k_{i}} \log \left(k_{i} N_{k_{i}}(y)^{2}\right) \leq \lim _{i \rightarrow+\infty} \frac{1}{k_{i}} \log \left(k_{i} \cdot 2^{2 k_{i} / i}\right)=0 .
\end{aligned}
$$

Thus $F$ contains the $z m$-set $N(y, U)$.

To prove the main result of the section we need the following result.

Lemma 3.6. - Let $(X, T)$ be a transitive system and $x \in \operatorname{Tran}_{T}$. Then $(X, T)$ is an E-system if and only if for each neighborhood $U$ of $x, N(x, U)$ has positive upper Banach density. 
Proof. - Suppose $(X, T)$ is an $E$-system. For each neighborhood $U$ of $x$, there exists an ergodic measure $\mu \in M(X, T)$ with $\mu(U)>0$. Take a generic point $y$ for $\mu$, then $N(y, U)$ has positive upper density. Moreover, $N(x, U)$ has positive upper Banach density as $x \in \operatorname{Tran}_{T}$.

Now we assume that for each neighborhood $U$ of $x, N(x, U)$ has positive upper Banach density. As $x \in \operatorname{Tran}_{T}$, one has $B D^{*}(N(x, V))>0$ for each non-empty open subset $V$ of $x$.

Let $\left\{V_{j}\right\}_{j=1}^{+\infty}$ be a countable basis of the topological space $X$. Put $U_{j}=\operatorname{cl}\left(V_{j}\right)$. As $B D^{*}\left(N\left(x, U_{j}\right)\right)>0$, there exist $a_{k}<b_{k}$ such that

$\lim _{k \rightarrow+\infty}\left(b_{k}-a_{k}\right)=+\infty, \lim _{k \rightarrow+\infty} \frac{1}{b_{k}-a_{k}}\left|N\left(x, U_{j}\right) \cap\left\{a_{k}, a_{k}+1, \ldots, b_{k}-1\right\}\right|>0$.

Now, set $\mu_{k}=1 /\left(b_{k}-a_{k}\right) \sum_{i=a_{k}}^{b_{k}-1} \delta_{T^{i} x}$. Let $\mu^{j}=\lim _{i \rightarrow+\infty} \mu_{k_{i}}$ be a limit point of $\left\{\mu_{k}\right\}_{k=1}^{+\infty}$ in the weak ${ }^{\star}$-topology. Clearly, $\mu^{j}$ is an invariant measure of $(X, T)$ and

$$
\begin{aligned}
\mu^{j}\left(U_{j}\right) \geq \lim _{i \rightarrow+\infty} \mu_{k_{i}}\left(U_{j}\right) & =\lim _{i \rightarrow+\infty} \frac{1}{b_{k}-a_{k}} \sum_{i=a_{k}}^{b_{k}-1} \delta_{T^{i} x}\left(U_{j}\right) \\
& =\lim _{i \rightarrow+\infty} \frac{\left|N\left(x, U_{j}\right) \cap\left\{a_{k}, a_{k}+1, \ldots, b_{k}-1\right\}\right|}{b_{k_{i}}-a_{k_{i}}}>0 .
\end{aligned}
$$

Let $\mu=\sum_{j=1}^{+\infty} \mu^{j} / 2^{j}$. Then $\mu$ is an invariant measure of $(X, T)$ and $\operatorname{Supp}(\mu)=X$. It follows from the transitivity of $(X, T)$ that $(X, T)$ is an $E$-system.

Now we are ready to show the main result of the section. Note that if $(X, T) \perp \mathcal{M}$, then $(X, T)$ is a weakly mixing system with dense set of minimal points (see [13]).

ThEOREM 3.7. - Let $(X, T)$ be a transitive TDS. If $(X, T) \perp \mathcal{M}_{0}$, then $(X, T)$ is a weakly mixing E-system.

Proof. - Let $x \in \operatorname{Tran}_{T}$ and let $U$ be a non-empty neighborhood of $x$. By Theorem 3.2, $N(x, U) \cap A \neq \varnothing$ for any $z m$-set $A$. This implies that $N(x, U)$ has non-empty intersection with any set whose lower Banach density is one, by Theorem 3.5. Thus $N(x, U)$ has positive upper Banach density. By Lemma 3.6, $(X, T)$ is an $E$-system.

Since $\mathcal{M}_{0}$ contains all minimal equicontinuous systems, $(X, T)$ is disjoint from all minimal equicontinuous systems. Moreover transitivity of $(X, T)$ implies weak disjointness from all minimal equicontinuous systems, which is the definition of weakly scattering property [12]. Since it is shown in [12] that a weakly scattering $E$-system is weakly mixing, $(X, T)$ is weakly mixing. 


\section{A transitive diagonal TDS with only one minimal point}

We have shown that any transitive diagonal TDS is in $\mathcal{M}_{0}^{\perp}$, and it is known that [12] if $(X, T)$ is in $\mathcal{M}^{\perp}$, then $(X, T)$ has a dense set of minimal points. In this section we will construct a transitive diagonal TDS with only one minimal point. Thus, the system is in $\mathcal{M}_{0}^{\perp} \backslash \mathcal{M}^{\perp}$. To start our construction we need some notations.

For $p \geq 2$ let $\Lambda_{p}=\{0,1, \ldots, p-1\}$ with the discrete topology, $\Sigma_{p}=\Lambda_{p}^{\mathbb{Z}_{+}}$ with the product topology and $\sigma: \Sigma_{p} \rightarrow \Sigma_{p}$ be the shift. For $n \geq 2$ and $a=(a(1), a(2), \ldots, a(n)) \in \Lambda_{p}^{n}$ (a block of length $n$ ), let $|a|=n, \sigma(a)=$ $(a(2), \ldots, a(n))$. We say a appears in $x=(x(1), x(2), \ldots) \in \Sigma_{p}$ or $x \in \Lambda_{p}^{m}$ with $m \geq n$ if there is $j \in \mathbb{N}$ with $a=(x(j), x(j+1), \ldots, x(j+n-1)$ ) (write $a<x$ for short) and we use $t^{i}$ to denote $t \ldots t(i$ times). For $b=(b(1), \ldots, b(m)) \in$ $\Lambda_{p}^{m}$, let $a b=(a(1), \ldots, a(n), b(1), \ldots, b(m)) \in \Lambda_{p}^{n+m}$. Denote $(i i \ldots)$ by $\boldsymbol{i}$, $0 \leq i \leq p-1$. Recall that a subset $S$ of $\mathbb{Z}_{+}$is thick, if for each $n \in \mathbb{N}$ there is $s_{n} \in S$ such that $S \supset\left\{s_{n}, s_{n}+1, \ldots, s_{n}+n\right\}$, and $S$ is piecewise syndetic, if it is the intersection of a thick set and a syndetic set. First we need the following lemma.

Lemma 4.1. - There is an E-system $(Y, \sigma)$ contained in $\left(\Sigma_{3}, \sigma\right)$ with a unique minimal point 0 such that $h_{\mathrm{top}}\left(\sigma,\left\{U_{1}^{c}, U_{2}^{c}\right\}\right)>0$, where $U_{i}=[i]_{Y}, i=1,2$.

Proof. - Choose a subset $A \subset \mathbb{Z}_{+}$with positive upper Banach density but being not piecewise syndetic. Let $Z_{1}^{\prime}=\operatorname{cl}\left(\operatorname{orb}\left(1_{A}, \sigma\right)\right) \subseteq \Sigma_{2}$. Then as $A$ has positive upper Banach density there is some $\sigma$-invariant ergodic measure $\mu$ on $\Sigma_{2}$ with $\mu([1])>0$. Let $Z_{1}=\operatorname{supp}(\mu)$ and $R_{1}=\sigma$. Then $\left(Z_{1}, R_{1}\right)$ is a nontrivial $E$-system. Since $A$ is not piecewise syndetic, $\left(Z_{1}, R_{1}\right)$ has $\mathbf{0}$ as its only minimal point.

Consider the space $Z_{2}=Z_{1} \times \Sigma_{2}$. It is clear that $W=\{\mathbf{0}\} \times \Sigma_{2}$ is a closed invariant subset of $Z_{2}$, and contains the set of minimal points of $R_{1} \times \sigma$. Collapsing $W$ to a point $p$ we get a factor $Z_{3}$ of $Z_{2}$, and let $\pi:\left(Z_{2}, R_{1} \times \sigma\right) \rightarrow$ $\left(Z_{3}, H\right)$ be the factor map. It is easy to see that $Z_{3}$ is a zero dimensional transitive system and has a unique minimal point $p$. Take $z_{1} \in Z_{1} \backslash\{\mathbf{0}\}$. Since $z_{1} \in \operatorname{supp}(\mu)$, by [7]

$$
E_{2}\left(Z_{2}, R_{1} \times \sigma\right) \supseteq\left\{\left(\left(z_{1}, y_{1}\right),\left(z_{1}, y_{2}\right)\right):\left(y_{1}, y_{2}\right) \in E_{2}\left(\Sigma_{2}, \sigma\right)\right\} .
$$

Let $w_{1}=\pi\left(z_{1}, \mathbf{0}\right)$ and $w_{2}=\pi\left(z_{1}, \mathbf{1}\right)$. Since any pair of two different points of $\Sigma_{2}$ is an entropy pair, we get $\left(w_{1}, w_{2}\right) \in E_{2}\left(Z_{3}, H\right)$. It is obvious that $w_{1}, w_{2} \neq p$. Take clopen neighborhoods $V_{i}$ of $w_{i}$ such that $V_{1} \cap V_{2}=\varnothing$ and $p \notin V_{1} \cup V_{2}$. Let $V_{0}=Z_{3} \backslash\left(V_{1} \cup V_{2}\right)$, then $V_{0}$ is also a clopen set and $p \in V_{0}$. 
Now we construct a map $\varphi: Z_{3} \rightarrow \Sigma_{3}$ with $\varphi(z)(i)=j$ if $H^{i}(z) \in V_{j}$. Let $Y=\varphi(Z)$. Then $\varphi:\left(Z_{3}, H\right) \rightarrow(Y, \sigma)$ is a factor map, and hence $(Y, \sigma)$ is an $E$-system with a unique minimal point $\varphi(p)=\mathbf{0}$.

Since $\left(\varphi\left(w_{1}\right), \varphi\left(w_{2}\right)\right) \in \varphi \times \varphi\left(E_{2}\left(Z_{3}, H\right)\right)$ and $\varphi\left(w_{1}\right) \neq \varphi\left(w_{2}\right)$,

$$
\left(\varphi\left(w_{1}\right), \varphi\left(w_{2}\right)\right) \in E_{2}(Y, \sigma) .
$$

It implies that $h_{\text {top }}\left(\sigma,\left\{U_{1}^{c}, U_{2}^{c}\right\}\right)>0$, since $\varphi\left(w_{i}\right) \in U_{i}$, where $U_{i}=[i]_{Y}$, $i=1,2$.

With the help of $(Y, \sigma)$, now we start our construction.

THEOREM 4.2. - There is a non-trivial diagonal transitive system having only one minimal point.

Proof. - The main idea of the example is that we construct a recurrent point $x \in \Sigma_{2}$ with the following two properties:

I) $(x, \sigma(x)) \in E_{2}(X, \sigma)$, where $X=\operatorname{cl}(\operatorname{orb}(x, \sigma))$;

II) for each $n \in \mathbb{N}, 0^{n}$ appears in $x$ syndetically.

It is clear that $(X, \sigma)$ is a diagonal transitive system having a unique minimal point $\mathbf{0}$. First we give the detailed construction of the recurrent point $x$.

Let $\phi: \mathbb{N} \longrightarrow \mathbb{N}$ such that $(\phi(1), \phi(2), \ldots)=(1,1,2,1,2,3,1,2,3,4, \ldots)$. Hence for each $k \in \mathbb{N}, \phi^{-1}(k)$ is infinite and $\phi(k) \leq k$ for each $k \in \mathbb{N}$.

Set $A_{1}=(10), n_{1}=\left|A_{1}\right|=2$. Let

$$
C_{0}^{1}=0^{2 n_{1}}, \quad C_{1}^{1}=A_{1} 0^{n_{1}} \quad \text { and } \quad C_{2}^{1}=\sigma\left(A_{1}\right) 00^{n_{1}} .
$$

Let $(Y, \sigma)$ be the system constructed in Lemma 4.1 and $y=(y(0), y(1), \ldots)$ be a transitive point of $(Y, \sigma)$. Since $(Y, \sigma)$ has a unique minimal point $\mathbf{0}$, there is $\ell_{1} \in \mathbb{N}$ such that $0^{n_{1}}$ appears in $y$ with gaps bounded by $\ell_{1}$. Let $b_{1}=2 \ell_{1} n_{1}$.

Set

$$
\begin{aligned}
& A_{2}=A_{1} 0^{n_{1}} C_{y(0)}^{1} \cdots C_{y\left(b_{1}-1\right)}^{1} 0^{2 n_{1}}, n_{2}=\left|A_{2}\right|, \\
& C_{0}^{2}=0^{2 n_{2}}, \quad C_{1}^{2}=A_{2} 0^{n_{2}}, \quad C_{2}^{2}=\sigma\left(A_{2}\right) 00^{n_{2}} .
\end{aligned}
$$

If $A_{1}, \ldots, A_{k}, C_{i}^{j}, i=0,1,2,1 \leq j \leq k$ and $b_{1}, \ldots, b_{k-1}$ are defined, we define inductively $A_{k+1}, C_{0}^{k+1}, C_{1}^{k+1}, C_{2}^{k+1}$ and $b_{k}$.

Since $(Y, \sigma)$ has a unique minimal point $\mathbf{0}$, there is $\ell_{k} \in \mathbb{N}$ such that $0^{n_{k}}$ appears in $y$ with gaps bounded by $\ell_{k}$. Let $b_{k}=2 \ell_{k} n_{k}$.

Set

$$
\begin{gathered}
A_{k+1}=A_{k} 0^{n_{k}} C_{y(0)}^{\phi(k)} C_{y(1)}^{\phi(k)} \cdots C_{y\left(b_{k}-1\right)}^{\phi(k)} 0^{2 n_{k}}, \quad n_{k+1}=\left|A_{k+1}\right|, \\
C_{0}^{k+1}=0^{2 n_{k+1}}, \quad C_{1}^{k+1}=A_{k+1} 0^{n_{k+1}}, \quad C_{2}^{k+1}=\sigma\left(A_{k+1}\right) 00^{n_{k+1}} .
\end{gathered}
$$

It is clear that $n_{k+1}=\left(4+2 \ell_{k} n_{\phi(k)}\right) n_{k}$.

TOME $135-2007-\mathrm{N}^{\mathrm{O}} 2$ 
Let $x=\lim _{k \rightarrow \infty} A_{k}$ and $X=\operatorname{cl}(\operatorname{orb}(x, \sigma)) \subset \Sigma_{2}$. It is clear that $x$ is a recurrent point of $\sigma$. We claim that $x$ satisfies I) and II).

I). - First we show $(x, \sigma(x)) \in E_{2}(X, T)$. Suppose that $V_{1}^{\prime}$ and $V_{2}^{\prime}$ are neighborhoods of $x$ and $\sigma(x)$ respectively. Then there is $k \in \mathbb{N}$ such that $V_{1}=\left[A_{k}\right] \subset V_{1}^{\prime}$ and $V_{2}=\left[\sigma\left(A_{k}\right) 0\right] \subset V_{2}^{\prime}$. Note that $V_{1} \cap V_{2}=\varnothing$, and consequently $V_{1} \subset V_{2}^{c}$ and $V_{2} \subset V_{1}^{c}$.

There are infinitely many $j$ such that $\phi(j)=k$. Thus, for those $j$

$$
A_{j+1}=A_{j} 0^{n_{j}} C_{y(0)}^{k} C_{y(1)}^{k} \cdots C_{y\left(b_{j}-1\right)}^{k} 0^{2 n_{j}} .
$$

Let $\mathcal{V}=\left\{V_{1}^{c}, V_{2}^{c}\right\}$ and $N_{k}=2 n_{k}$.

For $\ell \in \mathbb{N}$, let $r_{\ell}=N\left(\bigvee_{i=0}^{\ell-1} \sigma^{-i N_{k}}\left\{V_{1}^{c}, V_{2}^{c}\right\}\right)$ and $\left\{s_{1}, \ldots, s_{r_{\ell}}\right\} \subseteq\{1,2\}^{\ell}$ such that

$$
\bigcup_{m=1}^{r_{\ell}}\left(\bigcap_{i=0}^{\ell-1} \sigma^{-i N_{k}} V_{s_{m}(i)}^{c}\right)=X
$$

Now we claim $\bigcup_{m=1}^{r_{\ell}}\left(\bigcap_{i=0}^{\ell-1} \sigma^{-i} U_{s_{m}(i)}^{c}\right)=Y$, where $U_{i}, i=1,2$ were introduced in Lemma 4.1. If this were not true, then there is

$$
\omega \in Y \backslash \bigcup_{m=1}^{r_{\ell}}\left(\bigcap_{i=0}^{\ell-1} \sigma^{-i} U_{s_{m}(i)}^{c}\right) \text {. }
$$

Let $z \in\left[C_{\omega(0)}^{k} C_{\omega(1)}^{k} \cdots C_{\omega(\ell-1)}^{k}\right]$. Since $\omega \notin \bigcap_{i=0}^{\ell-1} \sigma^{-i} U_{s_{m}(i)}^{c}$ for each $m \in$ $\left\{1, \ldots, r_{\ell}\right\}$, there is $i_{m} \in\{0,1,2, \ldots, \ell-1\}$ such that $\omega\left(i_{m}\right)=s_{m}\left(i_{m}\right) \in\{1,2\}$. Hence $\sigma^{i_{m} N_{k}} z \in\left[C_{\omega\left(i_{m}\right)}^{k}\right] \subseteq V_{s_{m}\left(i_{m}\right)}$ and thus $\sigma^{i_{m} N_{k}} z \notin V_{s_{m}\left(i_{m}\right)}^{c}$. That is, $z$ is not in $\bigcap_{i=0}^{\ell-1} \sigma^{-i N_{k}} V_{s_{m}(i)}^{c}$ for each $m \in\left\{1,2, \ldots, r_{\ell}\right\}$. This implies that $z \notin \bigcup_{m=1}^{r_{\ell}}\left(\bigcap_{i=0}^{\ell-1} \sigma^{-i N_{k}} V_{s_{m}(i)}^{c}\right)$ which contradicts (2). Thus, by the claim we have

So, we have

$$
N\left(\bigvee_{i=0}^{\ell-1} \sigma^{-i}\left\{U_{1}^{c}, U_{2}^{c}\right\}\right) \leq r_{\ell}=N\left(\bigvee_{i=0}^{\ell-1} \sigma^{-i N_{k}}\left\{V_{1}^{c}, V_{2}^{c}\right\}\right)
$$

$$
\begin{aligned}
h_{\mathrm{top}}(\sigma, \mathcal{V}) & =\lim _{n \rightarrow+\infty} \frac{1}{n} \log N\left(\bigvee_{i=0}^{n-1} \sigma^{-i} \mathcal{V}\right) \\
& \geq \lim _{\ell \rightarrow+\infty} \frac{1}{\ell N_{k}} \log N\left(\bigvee_{i=0}^{\ell-1} \sigma^{-i N_{k}} \mathcal{V}\right) \geq \frac{1}{N_{k}} \lim _{\ell \rightarrow+\infty} \frac{1}{\ell} \log N\left(\bigvee_{i=0}^{\ell-1} \sigma^{-i N_{k}} \mathcal{V}\right) \\
& \geq \frac{1}{N_{k}} \lim _{\ell \rightarrow+\infty} \frac{1}{\ell} \log N\left(\bigvee_{i=0}^{\ell-1} \sigma^{-i}\left\{U_{1}^{c}, U_{2}^{c}\right\}\right)=\frac{1}{N_{k}} h_{\mathrm{top}}\left(\sigma,\left\{U_{1}^{c}, U_{2}^{c}\right\}\right)>0
\end{aligned}
$$

This implies that $(x, \sigma(x)) \in E_{2}(X, \sigma)$. 
II). - We now show that for each $n \in \mathbb{N}, 0^{n}$ appears in $x$ syndetically. It is suffices to prove that for each $k \in \mathbb{N}, 0^{n_{k}}$ appears in $x$ syndetically with gaps bounded by $2 b_{k}$.

Fix $k \in \mathbb{N}$. By the construction

$$
A_{k+1}=A_{k} 0^{n_{k}} C_{y(0)}^{\phi(k)} C_{y(1)}^{\phi(k)} \cdots C_{y\left(b_{k}-1\right)}^{\phi(k)} 0^{2 n_{k}} .
$$

As $0^{n_{k}}$ appears in $y$ with gaps bounded by $\ell_{k}, 0^{n_{k}}$ appears in

$$
C_{y(0)}^{\phi(k)} C_{y(1)}^{\phi(k)} \cdots C_{y\left(b_{k}-1\right)}^{\phi(k)}
$$

with gaps bounded by $2 n_{\phi(k)} \ell_{k} \leq 2 n_{k} \ell_{k}=b_{k}$. Thus $0^{n_{k}}$ appears in $A_{k+1}$ with gaps bounded by $b_{k}+n_{k} \leq 2 b_{k}$.

Assume that for $\ell \geq k+1,0^{n_{k}}$ appears in $A_{i}, k+1 \leq i \leq \ell$, with gaps bounded by $2 b_{k}$. Now we are going to prove that this is also true for $\ell+1$. First note that

$$
A_{\ell+1}=A_{\ell} 0^{n_{\ell}} C_{y(0)}^{\phi(\ell)} C_{y(1)}^{\phi(\ell)} \cdots C_{y\left(b_{\ell}-1\right)}^{\phi(\ell)} 0^{2 n_{\ell}} .
$$

If $\phi(\ell) \geq k$, then by the induction assumption and the construction of $C_{y(i)}^{\phi(\ell)}$ we know that $0^{n_{k}}$ appears in $A_{\ell+1}$ with gaps bounded by $2 b_{k}$. If $\phi(\ell)<k$, then by the induction assumption and the discussion similar to the case when $\ell=k+1$, we know that $0^{n_{k}}$ appears in $A_{\ell+1}$ with gaps bounded by $2 b_{k}$. Hence $0^{n_{k}}$ appears in $x$ syndetically with gaps bounded by $2 b_{k}$ since $x=$ $\lim _{m \rightarrow+\infty} A_{m}$.

A direct consequence of Theorem 4.2 is

Corollary 4.3. - There exists a TDS which is in $\mathcal{M}_{0}^{\perp}$ but not in $\mathcal{M}^{\perp}$.

Proof. - Let $(X, T)$ be the TDS constructed in Theorem 4.2. Then $(X, T)$ belong to $\mathcal{M}_{0}^{\perp}$ by [2]. As each TDS in $\mathcal{M}^{\perp}$ has a dense set of minimal points [13], we have that $(X, T) \in \mathcal{M}_{0}^{\perp} \backslash \mathcal{M}^{\perp}$.

To state another consequence of Theorem 4.2 we need some notions. Recall that a subset $S$ of $\mathbb{Z}_{+}$is thickly syndetic, if for each $n \in \mathbb{N}$ there is a syndetic set $\left\{s_{n}^{1}<s_{n}^{2}<\cdots\right\}$ such that $S \supset \bigcup_{n=1}^{\infty} \bigcup_{j=1}^{\infty}\left\{s_{n}^{j}, s_{n}^{j}+1, \ldots, s_{n}^{j}+n\right\} ; S$ is thick if it contains arbitrarily long intervals of natural numbers; $S$ is piece-wise syndetic if it is the intersection of a syndetic set with a thick set; and $S$ is an $m$-set if there are a minimal TDS $(X, T), x \in X$ and a non-empty open subset $U$ of $X$ such that $S \supset N(x, U)$. In [13] it is shown that each thickly syndetic set contains an $m$-set.

COROLlARY 4.4. - There is a thickly syndetic set which does not contain any $z m$-set. 
Proof. - Let $(X, T)$ be a non-trivial diagonal transitive system having only one minimal point and $x \in \operatorname{Trans}_{T}$. Then $(X, T) \in \mathcal{M}_{0}^{\perp}$ by [2]. If each thickly syndetic set contains a $z m$-set, then by Theorem 3.2, $N(x, U)$ is piecewise syndetic for any neighborhood $U$ of $x$. Hence $(X, T)$ has a dense set of minimal points by Lemma 2.1,1) in [13], which is a contradiction.

\section{TDS disjoint from $\mathcal{M}_{0}$, the general case}

In the previous sections we have discussed the problems assuming the transitivity. Now we study the properties of TDS disjoint from $\mathcal{M}_{0}$ without the assumption. We will show that if a $\operatorname{TDS}(X, T)$ is in $\mathcal{M}_{0}{ }^{\perp}$, then there exists an invariant measure $\mu$ with $\operatorname{Supp}(\mu)=X$.

We start with the following notation. Let $\Omega$ be a set of finite symbols, $\left\{y_{n}\right\}_{n \in \mathbb{N}} \in \Omega^{\mathbb{Z}_{+}}$and $k \in \mathbb{N}$. Let

$$
\begin{aligned}
& N_{k}\left(\left\{y_{n}\right\}_{n \in \mathbb{N}}\right)=\#\left\{u \in \Omega^{k}: \exists n \in N, i \in \mathbb{Z}_{+} \text {s.t. } u=y_{n}[i k ; i k+k-1]\right\}, \\
& B_{k}\left(\left\{y_{n}\right\}_{n \in \mathbb{N}}\right)=\#\left\{u \in \Omega^{k}: \exists n \in N, j \in \mathbb{Z}_{+} \text {s.t. } u=y_{n}[j ; j+k-1]\right\} .
\end{aligned}
$$

To study the problem in the general case we need to consider a sequence of $\mathcal{F}_{\text {lbd1 } 1 \text {-subsets. }}$

Definition 5.1. - A sequence $\left\{F_{n}\right\}_{1}^{\infty}$ of $\mathcal{F}_{\text {lbd1 }}$-subsets of $\mathbb{Z}_{+}$is uniform, if for any $\epsilon>0$ there exists $L_{\epsilon} \in \mathbb{N}$ such that for every integer interval $I$ with $|I| \geq L_{\epsilon}$ and every $n \in \mathbb{N}$ we have $\left|I \cap F_{n}\right| /|I| \geq 1-\epsilon$.

Let $a \in \Omega$ and $\left\{y_{n}\right\}_{n \in \mathbb{N}} \in \Omega^{\mathbb{Z}_{+}}$. Denote the set $\left\{z \in \Omega^{\mathbb{Z}_{+}}: z(0)=a\right\}$ by $[a]$. For a uniform sequence of $\mathcal{F}_{\text {lbd1 } 1 \text {-subsets we have }}$

LEMMA 5.2. - Let $F_{n}=\left\{i \in \mathbb{Z}_{+}: y_{n}(i)=a\right\}$. If $\left\{F_{n}\right\}_{n=1}^{+\infty}$ is a uniform sequence off $\mathcal{F}_{\mathrm{lbd} 1}$-subsets of $\mathbb{Z}_{+}$, then for $\epsilon>0$ there exists $N>0$ such that $N_{k}\left(\left\{y_{n}\right\}_{n \in \mathbb{N}}\right)<2^{\epsilon k}$ for $k \geq N$.

Proof. - Let $\sigma$ be the shift map on $\Omega^{\mathbb{Z}_{+}}$. Put

$$
Y=\operatorname{cl}\left(\bigcup_{n \in \mathbb{N}} \operatorname{orb}\left(y_{n}, \sigma\right)\right) \text {. }
$$

We have:

Claim. - $(Y, \sigma)$ has a unique $\sigma$-invariant measure $\delta_{(a, a, a, \ldots)}$.

Proof of Claim. - If the claim is not true, then there exists an ergodic measure $\mu$ of $(Y, \sigma)$ with $\mu \neq \delta_{(a, a, a, \ldots)}$. Take a generic point $y \in Y$ of $\mu$, then $y \neq(a, a, a, \ldots)$. Thus, there exists $b \in \Omega$ with $b \neq a$ and $y \in[b]$. 
Since $y$ is a generic point, $D^{*}(N(y,[b]))>0$. Let $\epsilon=\frac{1}{3} D^{*}(N(y,[b]))$. Since

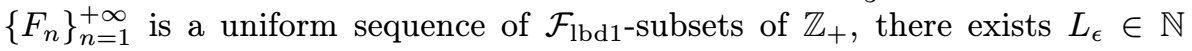
such that for every integer interval $I$ with $|I| \geq L_{\epsilon}$ and every $n \in \mathbb{N}$ we have $\left|I \cap F_{n}\right| /|I| \geq 1-\epsilon$.

Take $K \geq L_{\epsilon}$ with

$$
\frac{|N(y,[b]) \cap[0, K-1]|}{K} \geq 2 \epsilon .
$$

As $y \in \operatorname{cl}\left(\bigcup_{n \in \mathbb{N}} \operatorname{orb}\left(y_{n}, \sigma\right)\right)$, we can find $n \in \mathbb{N}$ and $\ell \in \mathbb{Z}_{+}$such that

$$
y[0, K-1]=\left(\sigma^{\ell} y_{n}\right)[0, K-1]
$$

We let $I_{\ell}=[\ell, \ell+K-1]$, then $\left|I_{\ell}\right| \geq L_{\epsilon}$ and $\left|I_{\ell} \cap F_{n}\right| /\left|I_{\ell}\right| \geq 1-\epsilon$. This implies that $\left|\left\{i \in \mathbb{Z}_{+}: y_{n}(i)=a, \ell \leq i \leq \ell+K-1\right\}\right| / K \geq 1-\epsilon$. Thus

$$
\frac{|N(y,[b]) \cap[0, K-1]|}{K} \leq 1-\frac{\left|\left\{i \in \mathbb{Z}_{+}: y_{n}(i)=a, \ell \leq i \leq \ell+K-1\right\}\right|}{K} \leq \epsilon,
$$

a contradiction to (3).

Hence we have $h_{\text {top }}(Y, \sigma)=0$ by the claim. Fix $\epsilon>0$. As in Lemma 3.4 there exists $N>0$ such that $N_{k}\left(\left\{y_{n}\right\}_{n \in \mathbb{N}}\right)<2^{\epsilon k}$ for $k \geq N$.

To prove the main result of the section we need to strengthen our Theorem 3.5 .

THEOREM 5.3. - Let $\left\{G_{n}\right\}_{n=1}^{+\infty}$ be a uniform sequence of $\mathcal{F}_{\text {lbd1 }}$-subsets of $\mathbb{Z}_{+}$. Then there is a minimal zero entropy system $(Y, \sigma) \subset\left(\Sigma_{2}, \sigma\right)$ such that for each $n \in \mathbb{N}$ there exists $y_{n} \in Y$ with $N\left(y_{n},[1]\right) \subset G_{n}$.

Proof. - We will modify the construction in Theorem 3.5. Let $f: \mathbb{N} \rightarrow \mathbb{N}$ be a map such that for each $i \in \mathbb{N}, f^{-1}(i)$ is infinite. Let $F_{n}=G_{f(n)}$ for each $n \in \mathbb{N}$. Then $\left\{F_{n}\right\}$ is a uniform $\mathcal{F}_{\text {lbd1 }}$-sets.

Step 1. - We will construct $A_{1}\left(k_{1}=\left|A_{1}\right|\right)$ and $F_{n}^{1} \subset F_{n}$ such that $A_{1}$ appears in $y_{n}^{1}=1_{F_{n}^{1}}$ with gaps bounded by $\ell_{1}$ and the first place where $A_{1}$ appears in $y_{n}^{1}$ is less than $\frac{l_{1}}{2}$ for each $n \in \mathbb{N}$ and $N_{k_{1}}\left(\left\{y_{n}^{1}\right\}_{n \in \mathbb{N}}\right)<2^{k_{1}}$.

Since $\left\{F_{n}\right\}$ is a uniform sequence of $\mathcal{F}_{\text {lbd1 }}$-sets, there exists $K_{1}>0$ such that $N_{k}\left(\left\{1_{F_{n}}\right\}_{n \in \mathbb{N}}\right)<2^{k}$ for $k \geq K_{1}$ by Lemma 5.2. Let $k_{1}=\max \left\{K_{1}, \min F_{1}+1\right\}$ and $A_{1}=1_{F_{1}}\left[0 ; k_{1}-1\right]$. Then $N_{k_{1}}\left(\left\{1_{F_{n}}\right\}_{n \in \mathbb{N}}\right)<2^{k_{1}}$. Set

$$
W_{n}^{1}=\left\{i \in \mathbb{Z}_{+}:\left[i k_{1} ; i k_{1}+k_{1}-1\right] \subset F_{n}\right\} .
$$

Choose $y_{n}^{1} \in \Sigma_{2}$ such that

$$
\begin{aligned}
& y_{n}^{1}\left[i k_{1} ; i k_{1}+k_{1}-1\right]=A_{1}, \quad i \in W_{n}^{1} \text { and } \\
& y_{n}^{1}\left[j k_{1} ; j k_{1}+k_{1}-1\right]=1_{F_{n}}\left[j k_{1} ; j k_{1}+k_{1}-1\right] \quad \text { if } j \notin W_{n}^{1} .
\end{aligned}
$$


Since $\left\{F_{n}\right\}$ is a uniform sequence of $\mathcal{F}_{\text {lbd1 }}$-sets, so is $\left\{W_{n}^{1}\right\}$. Hence we can find $\ell_{1}^{\prime} \in \mathbb{N}$ such that $W_{n}^{1}$ is syndetic with gaps bounded by $\ell_{1}^{\prime}$ for all $n \in \mathbb{N}$. Put $\ell_{1}=2 \ell_{1}^{\prime} \cdot k_{1}$. Then $N_{k_{1}}\left(\left\{y_{n}^{1}\right\}_{n \in \mathbb{N}}\right) \leq N_{k_{1}}\left(\left\{1_{F_{n}}\right\}_{n \in \mathbb{N}}\right)<2^{k_{1}}, A_{1}$ appears in $y_{n}^{1}$ with gaps bounded by $\ell_{1}$ and the first place which $A_{1}$ appears in $y_{n}^{1}$ is less than $\frac{1}{2} \ell_{1}$ for each $n \in \mathbb{N}$. If $F_{n}^{1} \subset \mathbb{Z}_{+}$satisfies $1_{F_{n}^{1}}=y_{n}^{1}$, then $F_{n}^{1} \subseteq F_{n}$.

Step 2. - We will construct $A_{m+1}$ and $y_{n}^{m+1} \in \Sigma_{2}$ such that

a) $A_{m+1}$ has the form of $A_{m} y_{m}^{m}\left[0, a_{m} k_{m}-1\right] A_{m}$.

b) $A_{i}$ appear in $y_{n}^{m+1}$ syndetically with gaps bounded by $\ell_{i}$ and the first place which $A_{i}$ appears in $y_{n}^{m+1}$ is less than $\frac{1}{2} \ell_{i}$ for each $1 \leq i \leq m+1$ and $n \in \mathbb{N}$.

c) $F_{n}^{m+1}=\left\{i \in \mathbb{Z}_{+}: y_{n}^{m+1}(i)=1\right\} \subset F_{n}$ for each $n \in \mathbb{N}$.

d) $N_{k_{i}}\left(\left\{y_{n}^{m+1}\right\}_{n \in \mathbb{N}}\right)<2^{\frac{1}{i} k_{i}}$ for each $1 \leq i \leq m+1$, where $k_{m+1}=\left|A_{m+1}\right|$.

Since $\left\{W_{n}^{m}\right\}$ is a uniform sequence of $\mathcal{F}_{\text {lbd1-sets }}$ and $A_{m}$ appears at places $k_{m} \cdot W_{n}^{m}$ in $y_{n}^{m}$, there exists $K_{m+1}>0$ such that $N_{k \cdot k_{m}}\left(\left\{y_{n}^{m}\right\}_{n \in \mathbb{N}}\right)<2^{k \cdot k_{m} / m+1}$ for $k \geq K_{m+1}$ by Lemma 5.2 .

Let $a_{m} \geq \max \left\{K_{m+1}, \ell_{1}, \ell_{2}, \ldots, \ell_{m}\right\}$ with $a_{m} \in W_{m}^{m}$. Set

$$
A_{m+1}=A_{m} y_{m}^{m}\left[0 ; a_{m} k_{m}-1\right] A_{m} \quad \text { and } \quad k_{m+1}=\left|A_{m+1}\right| \text {. }
$$

Then $k_{m} \mid k_{m+1}$ and $N_{k_{m+1}}\left(\left\{y_{n}^{m}\right\}_{n \in \mathbb{N}}\right)<2^{k_{m+1} / m+1}$. Set

$$
W_{n}^{m+1}=\left\{i \in \mathbb{Z}_{+}:\left[i k_{m+1} ; i k_{m+1}+k_{m+1}-1\right] \subset F_{n}\right\}
$$

Choose $y_{n}^{m+1} \in \Sigma_{2}$ such that

$$
\begin{aligned}
& y_{n}^{m+1}\left[i k_{m+1} ; i k_{m+1}+k_{m+1}-1\right]=A_{m+1} \text { for } i \in W_{n}^{m+1} \text { and } \\
& y_{n}^{m+1}\left[i k_{m+1} ; i k_{m+1}+k_{m+1}-1\right]=y_{n}^{m}\left[i k_{m+1} ; i k_{m+1}+k_{m+1}-1\right], i \notin W_{n}^{m+1} .
\end{aligned}
$$

As $y_{n}^{m+1}$ and $y_{n}^{m}$ differ possibly only at $\left[i k_{m+1} ; i k_{m+1}+k_{m+1}-1\right], i \in W_{n}^{m+1}$, we get

$$
F_{n}^{m+1}=\left\{i \in \mathbb{Z}_{+}: y_{n}^{m+1}(i)=1\right\} \subset F_{n}^{m} \cup \bigcup_{i \in W_{n}^{m+1}}\left[i k_{m+1} ; i k_{m+1}+k_{m+1}-1\right] \subset F_{n} .
$$

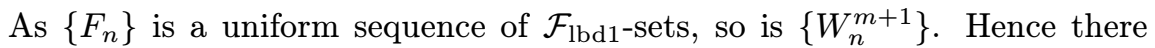
is $\ell_{m+1}^{\prime} \in \mathbb{N}$ such that $W_{n}^{m+1}$ is syndetic with gaps bounded by $\ell_{m+1}^{\prime}$ for each $n \in \mathbb{N}$. Let $\ell_{m+1}=2 \ell_{m+1}^{\prime} k_{m+1}$. Then $A_{i}$ appears in $y_{n}^{m+1}$ with gaps bounded by $\ell_{i}$ and the first place where $A_{i}$ appears in $y_{n}^{m+1}$ is less than $\frac{1}{2} \ell_{i}$ for each $1 \leq i \leq m+1$ and $n \in \mathbb{N}$ by the construction. It is also easy to see that $N_{k_{i}}\left(\left\{y_{n}^{m+1}\right\}_{n \in \mathbb{N}}\right) \leq N_{k_{i}}\left(\left\{y_{n}^{m}\right\}_{n \in \mathbb{N}}\right)<2^{k_{i} / i}$ for each $1 \leq i \leq m+1$. 
Let $y=\lim A_{m}$. By the construction, $A_{m}$ appears in $y$ with gaps bounded by $\ell_{m}$ for each $m \in \mathbb{N}$. That is, $y$ is a minimal point for the shift. Hence $(Y, \sigma)$ is minimal, where $Y=\operatorname{cl}(\operatorname{orb}(y, \sigma))$. As for each $i \in \mathbb{N}$

$$
N_{k_{i}}(y)=\lim _{m \rightarrow+\infty} N_{k_{i}}\left(A_{m}\right) \leq \limsup _{m \rightarrow+\infty} N_{k_{i}}\left(\left\{y_{n}^{m}\right\}_{n \in \mathbb{N}}\right)<2^{k_{i} / i}
$$

one has $\lim _{i \rightarrow+\infty} k_{i}^{-1} \log N_{k_{i}}(y)=0$.

For each $u \in B_{k}(y)$, there are $u_{1}, u_{2} \in N_{k}(y)$ such that $u<u_{1} u_{2}$. Thus $B_{k}(y) \leq k N_{k}(y)^{2}$ for any $k \in \mathbb{N}$ and hence

$$
h_{\mathrm{top}}(Y, \sigma)=\lim _{i \rightarrow+\infty} \frac{1}{k_{i}} \log B_{k_{i}}(y) \leq \lim _{i \rightarrow+\infty} \frac{1}{k_{i}} \log \left(k_{i} N_{k_{i}}(y)^{2}\right)=0 .
$$

Thus $(Y, \sigma)$ is a minimal system with zero entropy.

For each $n \in \mathbb{N}$ let $y_{n}$ be a limit point of $\left\{y_{i}^{i}\left[0 ; a_{i} k_{i}-1\right]\right\}_{f(i)=n}$. Let

$$
[1]=\{z \in Y: z(0)=1\} \text {. }
$$

Then we have

$$
N\left(y_{n},[1]\right) \subseteq \bigcup_{f(i)=n}\left\{j: y_{i}^{i}(j)=1,0 \leq j \leq a_{i} k_{i}-1\right\} \subseteq \bigcup_{f(i)=n} F_{i}^{i} \subseteq \bigcup_{f(i)=n} F_{i}=F_{n}^{\prime} .
$$

With the help of Theorem 5.3 we now prove the main result of the section.

THEOREM 5.4. - Let $(X, T)$ be a TDS in $\mathcal{M}_{0}{ }^{\perp}$, then there exists an invariant measure $\mu$ with $\operatorname{Supp}(\mu)=X$.

Proof. - First assume that $T$ is a homeomorphism and that there exists no invariant measure $\mu$ with $\operatorname{Supp}(\mu)=X$. Set $M=\operatorname{cl}\left(\bigcup_{\nu \in M(X, T)} \operatorname{Supp}(\nu)\right)$, and it is clear $M \neq X$. Hence there are a non-empty open set $U$ of $X$ and an open neighborhood $V$ of $M$ with $U \cap V=\varnothing$.

By Proposition 2.1(1), R(T) is dense in $U$. We may take a sequence of recurrent points $\left\{x_{n}\right\}_{n=1}^{+\infty} \subset U$ which is dense in $U$. Let $F_{n}=N\left(x_{n}, U^{c}\right) \supset$ $N\left(x_{n}, V\right), n=1,2, \ldots$

Claim. $-\left\{F_{n}\right\}_{n=1}^{+\infty}$ is a uniform sequence of $\mathcal{F}_{\text {lbd1 }}$-subsets of $\mathbb{Z}_{+}$.

Proof of claim. Assume $\left\{F_{n}\right\}_{n=1}^{+\infty}$ is not a uniform sequence of $\mathcal{F}_{\text {lbd1-subsets }}$ of $\mathbb{Z}_{+}$. Then there exists $\epsilon>0$ such that for any $k \in \mathbb{N}$ we can find an integer interval $I_{k}$ and $n_{k} \in \mathbb{N}$ with $\left|I_{k}\right| \geq k$ and $\left|F_{n_{k}} \cap I_{k}\right| /\left|I_{k}\right|<1-\epsilon$, i.e., $\left|N\left(x_{n_{k}}, U\right) \cap I_{k}\right| /\left|I_{k}\right| \geq \epsilon$. 
Let $\mu_{k}=\frac{1}{\left|I_{k}\right|} \sum_{i \in I_{k}} \delta_{T^{i} x_{n_{k}}}$, and $\mu=\lim _{i \rightarrow+\infty} \mu_{k_{i}}$ be a limit point of $\left\{\mu_{k}\right\}_{k=1}^{+\infty}$ in the weak ${ }^{\star}$-topology. Clearly, $\mu$ is an invariant measure of $(X, T)$ and

$$
\begin{aligned}
\mu\left(V^{c}\right) & \geq \lim _{i \rightarrow+\infty} \mu_{k_{i}}\left(V^{c}\right)=\lim _{i \rightarrow+\infty} \frac{1}{\left|I_{k_{i}}\right|} \sum_{j \in I_{k}} \delta_{T^{j} x_{n_{k_{i}}}}\left(V^{c}\right) \\
& \geq \lim _{i \rightarrow+\infty} \frac{\left|N\left(x_{n_{k_{i}}}, U\right) \cap I_{k_{i}}\right|}{\left|I_{k_{i}}\right|} \geq \epsilon .
\end{aligned}
$$

On the other hand, we have $\mu\left(V^{c}\right) \leq \mu\left(M^{c}\right)=0$ by the definition of $M$, which leads to a contradiction. This ends the proof of the claim.

By the above claim and Theorem 5.3, there is a minimal system $(Y, \sigma) \subset$ $\left(\Sigma_{2}, \sigma\right)$ such that for each $n \in \mathbb{N}$ there exists $y_{n} \in Y$ with $N\left(y_{n},[1]\right) \subset F_{n}$. Let

$$
J=\operatorname{cl}\left(\bigcup_{i=0}^{+\infty} \bigcup_{n=1}^{+\infty}(T \times \sigma)^{i}\left(x_{n}, y_{n}\right)\right) \bigcup\left(X \backslash \bigcup_{i=-\infty}^{+\infty} T^{i} U\right) \times Y .
$$

As $\left\{x_{n}: n \in \mathbb{N}\right\}$ is dense in $U$ and each $x_{n}$ is recurrent, we have

$$
\bigcup_{i=-\infty}^{+\infty} T^{i} U \subset \operatorname{cl}\left(\bigcup_{i=0}^{+\infty} \bigcup_{n=1}^{+\infty} T^{i} x_{n}\right)
$$

Thus $J$ is a joining of $X$ and $Y$, and hence $J=X \times Y$.

It is easy to see that $\operatorname{cl}\left(\bigcup_{i=0}^{+\infty} \bigcup_{n=1}^{+\infty}(T \times \sigma)^{i}\left(x_{n}, y_{n}\right)\right) \supset U \times[1]$. As $U \times[1]$ is open, we have

$$
\left(\bigcup_{i=0}^{+\infty} \bigcup_{n=1}^{+\infty}(T \times \sigma)^{i}\left(x_{n}, y_{n}\right)\right) \cap(U \times[1]) \neq \varnothing .
$$

Therefore, there exist $i$ and $n$ with $\left(T^{i} x_{n}, \sigma^{i} y_{n}\right) \in U \times[1]$, i.e., $N\left(x_{n}, U\right) \cap$ $N\left(y_{n},[1]\right) \neq \varnothing$, which contradicts the fact that $N\left(y_{n},[1]\right) \subset F_{n}=N\left(x_{n}, U^{c}\right)$.

If $T$ is not a homeomorphism, we pass to the natural extension and observe that an invariant measure with full support are mapped to an invariant measure with full support by factor maps.

\section{BIBLIOGRAPHY}

[1] F. Blanchard - "Fully positive topological entropy and topological mixing", in Symbolic dynamics and its applications (New Haven, CT, 1991), Contemp. Math., vol. 135, Amer. Math. Soc., 1992, p. 95-105. , "A disjointness theorem involving topological entropy", Bull. Soc. Math. France 121 (1993), p. 465-478.

[3] F. Blanchard, B. Host \& A. MaAss - "Topological complexity", Ergodic Theory Dynam. Systems 20 (2000), p. 641-662. 
[4] F. Blanchard \& Y. Lacroix - "Zero entropy factors of topological flows", Proc. Amer. Math. Soc. 119 (1993), p. 985-992.

[5] H. Furstenberg - "Disjointness in ergodic theory, minimal sets, and a problem in Diophantine approximation", Math. Systems Theory 1 (1967), p. $1-49$.

[6] _ Recurrence in ergodic theory and combinatorial number theory, Princeton University Press, 1981, M. B. Porter Lectures.

[7] E. GLASNER - "A simple characterization of the set of $\mu$-entropy pairs and applications", Israel J. Math. 102 (1997), p. 13-27.

[8] E. Glasner \& B. Weiss - "Quasi-factors of zero-entropy systems", J. Amer. Math. Soc. 8 (1995), p. 665-686.

[9] _ "Locally equicontinuous dynamical systems", Colloq. Math. 84/85 (2000), p. 345-361, Dedicated to the memory of Anzelm Iwanik.

[10] W. H. HE \& Z. L. ZHOU - "A topologically mixing system whose measure center is a singleton", Acta Math. Sinica (Chin. Ser.) 45 (2002), p. 929-934 (Chinese).

[11] W. Huang, S. Shao \& X. YE - "Mixing via sequence entropy", Comtemp. Math. 385 (2005), p. 101-122.

[12] W. HuAng \& X. YE - "Generic eigenvalues, generic factors and weak disjointness", Preprint.

[13] , "Dynamical systems disjoint from any minimal system", Trans. Amer. Math. Soc. 357 (2005), p. 669-694.

[14] W. PARRY - "Zero entropy of distal and related transformations", in Topological Dynamics (Symposium, Colorado State Univ., Ft. Collins, Colo., 1967), Benjamin, New York, 1968, p. 383-389.

[15] B. Song \& X. YE - "A minimal completely positive, non uniformly positive entropy example", to appear in J. Difference Equations and Applications.

[16] B. WEISS - "Topological transitivity and ergodic measures", Math. Systems Theory 5 (1971), p. 71-75. 